\title{
Appendix 4: Template of Information for Clients
}

\author{
(C) 2012 Mark Widdowson
}

\author{
The Process and Outcome of \\ Transactional Analysis Psychotherapy for \\ the Treatment of Depression - Information \\ for Participating Clients
}

\begin{abstract}
About the Research
We are investigating transactional analysis (TA) psychotherapy, and in particular how it can be used in the treatment of depression. Transactional Analysis is widely used in psychotherapy in the UK and in Europe. TA therapists report good results from using TA with a wide range of clients, although there has been little formal research into TA therapy. This research project is exploring the processes and outcomes of TA therapy in the treatment of depression. Previous research suggests that the different types of therapy are roughly equivalent to each other in terms of effectiveness, and we anticipate finding that TA has outcomes which are equal to other types of therapy. TA shares many theories and methods with other types of therapy which are known to be effective which is why we feel confident in predicting generally good outcomes.
\end{abstract}

All the therapists participating in this research are trained and qualified in TA psychotherapy and work actively and respectfully with clients to help them explore their experiences; this enables clients to make sense of them and to help them make changes in their life through the development of a collaborative working relationship. In general, therapy differs from other ways of helping in that it refrains from giving advice but encourages clients to find their own solutions to their problems and supports them in achieving these.

Other goals of this research are:

1. Improving the training and effectiveness of TA therapists by teaching them how to integrate the findings of the research into therapy and therapy training courses and developing better ways of studying psychotherapy.
2. Improving the effectiveness of therapists through understanding the ways in which therapeutic change takes place and in refining how therapists deliver therapy. Other specific research projects may be developed but you will receive additional information about these if you are asked to take part.

If you are eligible for this study and are willing to take part, you will be offered TA psychotherapy from the therapist you have contacted.

\section{Who is doing the research?}

The principal researcher is Mark Widdowson, MSc (TA Psychotherapy), Teaching and Supervising Transactional Analyst, UKCP Registered Psychotherapist. Mark is a Ph $D$ student at the University of Leciester and is investigating the process and outcome of TA psychotherapy in the treatment of depression for his doctoral research. The whole research is being overseen by Professor Sue Wheeler at the University of Leicester, and Professor John McLeod at the University of Abertay, Dundee. If you have any concerns or queries about the research you may contact Mark directly either via e-mail at —or by telephone by calling

The research has been approved by the Research Ethics Committee at the University of Leicester and follows the research ethics guidelines of the British Association for Counselling and Psychotherapy (BACP).

\section{Complaints}

If you wish to make a complaint about the therapy or the research you may contact Mark, the principal researcher directly or you may contact Professor Wheeler at the University of Leicester. Her e-mail address is

\section{About the Therapists}

All the therapists participating in this study are professionally registered, trained and experienced therapists each with a minimum of five years training and over 750 hours of experience in working with clients. The therapists have been carefully selected to 
ensure that you will be receiving good quality therapy from a properly trained and experienced therapist.

All the therapists who will be providing the therapy are professionally registered members of professional counselling and psychotherapy organisations and have professional indemnity insurance and abide by the codes of ethics and practice of their professional organisations. As qualified TA therapists, all the therapists are registered with the United Kingdom Council for Psychotherapy. The therapist you are working with will give you more information regarding any additional organisations they are affiliated to.

All UKCP registered therapists, regardless of their level of training or experience must have regular clinical supervision where they discuss their work and their case load. The therapists participating in this study will be receiving regular supervision, which will help monitor their work and to provide a safeguard as to the quality of the therapy that you will receive.

What will I be required to do for this study?

The number of therapy sessions will be agreed between the therapist and yourself during the first few weeks of therapy. This agreement will form part of a therapy contract that will be reviewed regularly. You can be offered up to a maximum of 16 weekly sessions of 50 minutes. Previous research suggests that 16 sessions of therapy is sufficient for most people with mildmoderate depression to obtain significant relief from their symptoms.

In the course of the study, we will ask you to give us information about your therapy, including your perceptions of your problems and how you are functioning, as well as your experience of specific therapy sessions.

We will ask you to fill out four short questionnaires each week, and to have your sessions audio recorded. You will need to allow extra time both before and after your sessions to fill out the questionnaires. The time needed for filling these out will usually be around 20 minutes in total each week.

At the end of the first, third and sixth sessions you will also be asked to complete an additional short questionnaire relating to how you are experiencing your therapist and how you are working together. This will take less than ten minutes to complete.

In addition, after every eight sessions, at the end of treatment, and at two follow up interviews after your therapy has finished, we will ask you to fill out more questionnaires and be interviewed by a member of the research team about your experience of therapy. The researcher who will conduct the follow up interviews will also be a qualified therapist.
The point of all this is to help us discover information that may be useful for developing and evaluating TA psychotherapy in routine practice and specifically in the treatment of depression, and to improve the training of TA psychotherapists.

Two of the questionnaires that are used every eight sessions are included in this information pack. If you are interested in participating in the therapy, please fill these out and bring them along to the first session with your therapist. These forms will enable us to track the changes you make as a result of the therapy.

This research involves several stages:

1. First, after making contact with your therapist, your therapist will have invited you to attend a preliminary intake interview session. This is normal procedure for beginning therapy and the therapist will have discussed the option of participation in this research in the interview. The intake interviews normally take around one hour, although your therapist will have advised you as to their usual procedure for these intake interviews. The main purpose of this session is for us to make sure that therapy is appropriate for you, and to give you some information about the research.

Your therapist will ask you some questions about: the kinds of problems you are currently having; your current relationships and employment details; problems you have had in the past; and your personal history (including details of the family you grew up in), to make sure that they can help you or that you do not have some other condition that indicates the need for a different approach.

For the purposes of the research, we will not be able to see you if you are currently in psychotherapy or counselling elsewhere or if you are on antidepressant medication. You will not be suitable to take part in this research if you are going through current severe substance misuse, active psychotic condition or current domestic violence. In these cases, you will be advised of the options available to you for accessing therapy.

If you are interested in participating in the research, you will then be asked to read this information sheet and to sign the consent form. Please read over this information and the consent form carefully and make sure you understand it; note anything that may be unclear or that may be of concern to you, so you can discuss it with your therapist; do not sign the consent form yet.

If you decide you would like to participate and if you fit our guidelines, you will be asked to sign the consent form, and to complete some additional questionnaires prior to your first therapy session. Participation in the research is entirely optional and if you decide not to participate, you will still be able to access therapy. 
You will be given an information sheet on how you can get the most out of therapy, which also discusses some of the things you can expect to happen in the sessions.

2. In the study, you will work with your therapist up to a maximum of 16 sessions; the specific amount will be agreed by you and your therapist during the first few weeks of therapy. Together you will agree a therapy contract which will be your working agreement with your therapist about the nature of your therapy and the focus of your therapy. You will meet with your therapist once each week for 50 minutes.

\section{Each of these sessions will be audio recorded}

Immediately before and after each session, you will be asked to fill out brief questionnaires about how you are doing or about your experience of the session. The completion of the questionnaires should take about 20 minutes each week.

At the end of each session, you will complete a short questionnaire which is used to evaluate the session. If you feel the session was good, we want to know why it was good and what made it good. If you feel the session wasn't so good, we also want to know why it wasn't so good and what could have been different. During the data analysis phase of the research we may compare your session evaluations with a transcript of the session, to see if we can identify important and effective features of TA therapy and to help us learn more about what can be improved.

In entering into a therapy contract, you will be asked to commit to attending sessions regularly and to avoid cancelling at short notice wherever possible. Your therapist will advise you of the procedure for cancellation or rearrangement of sessions.

3. At the end of therapy and at a follow-up session, you will meet with a member of the research team (this will be someone else and not the therapist you have worked with), who will interview you about your problems and your experience of therapy, and ask you to complete some additional questionnaires. This should take about one to one and a half hours each time.

\section{CONFIDENTIALITY}

We routinely use audio recordings for the purposes of supervision and for the research, and in the consent form we are asking for your permission for that. We will separately ask you to give us permission to keep the recordings of your sessions and research interviews for research purposes, including training other therapists. Because it is important for us to protect your confidentiality, we will be taking several precautions:
- First of all, we will be using codes instead of names to identify all of the recordings and questionnaires.

- In addition, we will edit your name and any other identifying information from any transcripts we might make of parts of your sessions.

- We will disguise any information we might record in transcripts, notes, case studies or in material for publication when describing your case, for example, your profession, age, marital status, number of children and so on might be changed to help conceal your identity and reduce the chances that you could be identified in any way.

- The recordings will be stored on a passwordprotected computer, and back-ups will be stored in locked filing cabinets.

- Only your therapist, their supervisor and approved research staff will be allowed to have access to these recordings.

- Unless you tell us otherwise, questionnaires and recordings will be separated from your personal details and kept for a maximum of 5 years, providing there is scientific reason to do so, by the principal researcher and the research team. Questionnaires will be destroyed and recordings will be erased when there is no longer any scientific use for this data. We will review these issues with you after every eight sessions and again at the end as part of the follow up interview process.

- There are some situations that can arise in which we may have to take action to protect you or someone else from harm and have to reveal information that has come to light in interviewing a participant in this study or during therapy sessions. An example is where information was revealed that there was a child being abused by someone. If such a situation arises, we would limit the disclosure to what is absolutely necessary. We would also make every effort to fully discuss it with you beforehand. Your therapist will advise you of their policies and procedures regarding confidentiality.

POSSIBLE RISKS AND WHAT TO DO ABOUT THEM Before you consent to take part in this study, we want you to know about the possible risks of doing so, and how you can reduce those risks.

\section{Self-consciousness about being recorded.}

Although most people in the past have been able to disregard the recording equipment, a few have felt inhibited or self-conscious and have found it difficult to talk about deeply personal matters. If you think being 
audio recorded will interfere with your receiving help in therapy, please do not volunteer for this study. Audio recording is valuable for research and supervision purposes and to help us find out more about the things we are investigating. You may have concerns about the recordings or what will happen to them - please see below for more information. If you have any outstanding queries, you may discuss these with your therapist, or with the principal researcher.

\section{Getting bored with all the forms.}

There are a lot of forms to fill out for this research, and some people find them tedious and boring. Most clients find them interesting and a helpful addition to their therapy which helps them to reflect on and account for the changes that they are making. Please do not volunteer for this research if you hate filling out forms.

\section{Getting worse.}

Most clients experience temporary emotional discomfort or distress during therapy, including strong emotions as a natural part of the process. The therapist will work actively with you to help you deal with any painful emotions that may surface. If, however, you are seriously concerned about this, you may wish to reconsider volunteering for this study. If you volunteer and problems do occur, please report them to your therapist, who will do their best to address the difficulty. It may even turn out that the therapy is either not helping or, in rare instances, is causing harm; in such cases, it may be necessary to stop therapy or to refer you to a different form of treatment.

\section{Not getting better}

It is also possible that, at the end of your treatment, you may be in need of further therapy. If you feel you need further treatment, you and your therapist can discuss possible options. For example, they may offer you a referral to another therapist, type of therapy, or agency. This discussion will begin well in advance of your agreed ending date and will not be left until the end, so you will have time to prepare.

Starting therapy can be challenging and we recognise that things can happen that make it seem difficult to carry on with therapy. You are free to leave at any stage. We do, however, stress that it can be helpful for you to take the chance to discuss any difficulties with your therapist or one of the research team so we can address directly any problems that you raise.

\section{POTENTIAL BENEFITS}

In contrast to the risks listed above, there may also be some direct and indirect benefits for you or other people if you choose to take part in this study:

- As a result of the treatment, you are likely to feel better and less bothered by the problems you have been having. Previous research suggests that most clients experience significant improvement through therapy.
- Previous clients have reported that completing the research questionnaires and interviews helped them to get more out of their treatment. These procedures may also help you learn things about yourself.

- $\quad$ As you will be completing questionnaires at each session your progress will be closely monitored and evaluated and the therapy you will receive will be refined to increase the benefits you will obtain from the therapy.

Finally, you will be helping us better understand how TA therapy works, and in particular how we can use TA therapy in the treatment of depression. The research will also help psychotherapists develop better ways of helping other people, and assist us in training our post graduate students.

What notes are kept?

Your therapist will make some notes after each session, which will record the themes and issues that you both discussed in the session, what they did (what interventions they made and what theories and methods they used), and how you both seemed to be working together.

Your therapist will not be keeping detailed notes relating to specific events from your life and will keep your notes in such a way that your anonymity is preserved (see below). All notes will be stored securely in a locked cabinet and a code will be used instead of your name. Only your therapist and the principal researcher will know who the codes relate to.

The notes are firstly to help your therapist monitor and review your work together and secondly for research purposes. The notes will be used in the research to help us identify how therapists understand and work with particular themes or issues and also to see if we can identify common themes which affect people with depression. It is possible we may find that different therapists work differently with similar issues or problems, and we want to know why, what influences their way of working, and also what the outcome is of different ways of working.

You are entitled to see any notes kept about you and you can request a copy of your notes from your therapist. If you have any concerns or queries about the notes which are kept about you, you can discuss this with your therapist and/or the principal researcher.

Why will the therapy sessions be audio-recorded and what will happen to the recordings?

All of the therapy sessions will be audio-recorded. The recordings of sessions will potentially be used for several purposes:

- Your therapist might listen to segments of sessions for the purposes of reviewing the work as a part of their routine reflection and review on their work. 
- Your therapist might play excerpts of the recording in their clinical supervision. All therapists, regardless of their experience, have regular on-going supervision, which helps ensure the quality of the therapy you will receive. This will help your therapist to refine their work with you. Any extracts that your therapist might play in supervision will not include details which might identify you.

- Some sessions, or some extracts from sessions, will be transcribed (typed up) for the purposes of researching the process of therapy.

- No names or places will be included in the transcripts, and all details which might lead you, someone else or a particular place, to be identified will be omitted to preserve your anonymity and ensure that no one who might read any transcript could recognise you or someone else.

- Only your therapist, their supervisor and up to two members of the research team will listen to any recordings of the sessions. This means only professionals involved either directly or indirectly in your therapy will hear any part of the recordings.

- Once the research has finished, the recordings will be destroyed.

- $\quad$ All recordings will be stored securely until they are destroyed

What will happen to the transcripts?

The transcripts are an important part of the research process. Transcripts of sessions or segments of sessions will be analysed by the researcher to help our research into the therapy process. Some anonymised transcripts may be included in the $\mathrm{Ph} D$ thesis of the principal researcher. Some suitably disguised and anonymised transcripts may be used in professional publications. Some of the therapy cases will be written up as case studies which will be used to help us understand the therapy process in more depth. You will be asked at the end of your therapy if you are willing for a case study to be written about you. Your identity will be heavily concealed in any material which is written about you to preserve your anonymity.

What are the follow-up Interviews and why are they being done?

A member of the research team will contact you after you have finished therapy, and several months after the end of the therapy to arrange an interview with you to evaluate your experience of therapy. We want to hear honest feedback about the therapy process and your experiences of therapy and of being part of the research. This will help us to understand more about:

- How people change throughout therapy

- Which aspects of the therapy have been most helpful to you

- How we can improve therapy

You will also be asked to complete some additional questionnaires so we can evaluate your progress. The interview and completing the questionnaires should take about one to one and a half hours each time.

Thank you for your interest in this research. 